\title{
Time-dependent Photodynamic Therapy for Multiple Targets: A Highly Efficient AIE-active Photosensitizer for Selective Bacterial Elimination and Cancer Cell Ablation
}

\author{
Qiyao Li, ${ }^{\dagger, \#}$ Ying Li, ${ }^{\dagger,+, \#}$ Tianliang Min, ${ }^{+}$Junyi Gong, ${ }^{\dagger}$ Lili Du, ${ }^{\dagger}$ Kai Li, ${ }^{+}$David Lee Phillips, ${ }^{\dagger}$ Junkai \\ Liu, ${ }^{\dagger}$ Jacky W. Y. Lam, ${ }^{\dagger}$ Herman H. Y. Sung, ${ }^{\dagger}$ Ho Chun Loong, ${ }^{+}$Ian D. Williams, ${ }^{\dagger}$ Ryan T. K. Kwok, ${ }^{\dagger}$ \\ Jianguo Wang* ${ }^{*}, \perp$ and Ben Zhong Tang* ${ }^{\dagger},+, \S, \|$
}

\begin{abstract}
${ }^{\dagger}$ Department of Chemistry, Hong Kong Branch of Chinese National Engineering. Research Center for Tissue Restoration and Reconstruction, Institute of Molecular Functional Materials, State Key Laboratory of Molecular Nanoscience, Division of Life Science and Department of Chemical and Biomedical Engineering, The Hong Kong University of Science and Technology, Clear Water Bay, Kowloon, Hong Kong, China

* Center for AIE Research, College of Materials Science and Engineering, Shenzhen University, Shenzhen 518060, China

${ }^{+}$Department of Biomedical Engineering, Southern University of Science and Technology, Shenzhen, Guangdong 518055, China

${ }^{\ddagger}$ Department of Chemistry, The University of Hong Kong, Pokfulam Road, Hong Kong, China

${ }^{\perp}$ College of Chemistry and Chemical Engineering, Inner Mongolia University, Hohhot 010021, China

${ }^{\S}$ HKUST Shenzhen Research Institute, No. 9 Yuexing $1^{\text {st }}$ Rd, South Area, Hi-tech Park, Nanshan, Shenzhen 518057, China

" Center for Aggregation-induced Emission, SCUT-HKUST Joint Research Institute, State Key Laboratory of Luminescent Materials and Devices, South China University of Technology, Guangzhou 510640, China.
\end{abstract}

\begin{abstract}
Pathogen infection and cancer are the two major human wealth and health problems. Fluorescence-guided photodynamic therapy (FL-PDT) emerged as a hotspot in antibacterial and anticancer treatment with prominent merits including noninvasiveness, no drug resistance, real-time monitoring, spatiotemporal precision and synergistic effect over conventional therapeutic modalities. In this work, we achieved successfully an organic salt photosensitizer (PS), called 4TPA-BQ with aggregation-induced emission feature via one-step reaction. Owing to its aggregation-induced reactive oxygen species generation effect and sufficient small energy gap between the singlet and triplet states, 4TPA-BQ shows a satisfactorily high ${ }^{1} \mathrm{O}_{2}$ generation efficiency of $98.7 \%$. Unprecedentedly, by regulating the external conditions, time-dependent, specific and controllable targeting via FL-PDT was realized for the first time. Both in vitro and in vivo experiments confirmed that 4TPA-BQ exhibited potent photodynamic antibacterial performance even against ampicillin-resistant Escherichia coli with good biocompatibility in a short time (15 min), indicating that 4TPA-BQ holds promising potential for combating multiple pathogen bacteria. When the incubation time persisted long enough to $12 \mathrm{~h}$, cancer cells were ablated efficiently while the normal cells were essentially unaffected. This is the first attempt to explore a novel time-dependent PDT with the aid of one individual PS for bacterial elimination and cancer cell ablation, which not only update the design principle of efficient PSs, but also stimulates FL-PDT development for potential clinical applications.
\end{abstract}

\section{INTRODUCTION}

Pathogen infections cause severe illness and significant mortality globally. During the past decades, antibiotics have been employed predominately to treat diseases caused by bacteria since the first discovery of penicillin in 1928 . However, they suffer from confined targets and common side effects such as diarrhea, nausea and upset stomach. Additionally, after a long period abuse of antibiotics, the emergence of drug-resistant bacteria has posed a severe threat to human health. A recent study reported that about $70 \%$ normal $E$. coli became drug resistant strains only after $3 \mathrm{~h}$ exposure to antibiotics. ${ }^{1}$ Currently, we are confronted with an intractable situation that new antibiotics development fails to keep pace with the generation of drug-resistant bacteria. Therefore, developing alternative antibacterial therapeutics is of utmost urgency and great importance to clinical application.

Cancer, meanwhile, is another major public health problem. Globally, about 1 in 6 deaths is due to cancer. In 2019, about $1,762,450$ new cancer cases will be diagnosed and there will be 606,880 cancer deaths in the United States. ${ }^{2}$ To date, although various therapeutic modalities such as surgery, chemotherapy and radiotherapy have been exploited, they show different problems. ${ }^{3,4}$ Specifically, surgery operation is invasive to human body and it is hard to ablate tumors completely and precisely without causing harm to healthy tissues. Chemotherapy drugs, such as Cisplatin can lead to nephrotoxicity, increased risk to get infection and drug resistance. Patients who receive radiotherapy treatment, on the other hand, may suffer from fatigue and loss of hair in the treatment area. 
Recently, photodynamic therapy (PDT) has emerged as a promising method to combat pathogen bacteria as well as a powerful clinical protocol for cancer treatment. ${ }^{5-9}$ PDT employs photosensitizers (PSs) to produce destructive singlet oxygen $\left({ }^{1} \mathrm{O}_{2}\right)$ or other reactive oxygen species (ROS) from endogenous molecular oxygen under light irradiation. ${ }^{10}$ Thus, combing the merits of PS and light irradiation, PDT exhibits several conspicuous advantages including noninvasiveness, no drug resistance, low cytotoxicity, selective targeting, spatiotemporal precision and synergistic effect over conventional therapeutic modalities. Among major achievements on PDT advance, fluorescenceguided PDT (FL-PDT), with the unique advantage of real-time monitoring, has attracted increasing attention. PS, which serves as the key component of FL-PDT, plays a decisive role in therapeutic effect. ${ }^{11,12}$ Most conventional organic PSs, however, encounter the problem of aggregation-caused quenching (ACQ) in biosystems, resulting in undesired weak emission and poor ROS generation. Luckily, the discovery of the aggregation-induced emission (AIE) phenomenon offers a convenient solution. ${ }^{13-19}$ Unlike ACQ luminophores, AIE luminogens are weakly emissive in dilute solutions. However, they show strong emission and ROS generation in the aggregate state, owing to the mechanism of restriction of intramolecular motion (RIM). ${ }^{20,21}$ Thus, AIE-based PSs hold promising potential in PDT applications. Although research on AIE-based PSs in anticancer and antibacterial treatments proceeds rapidly, various problems remain to be solved. One common problem is that to realize selective binding, specific targeting ligands are needed to introduce to PSs. Such a method is not only tedious in synthesis but also of high cost. ${ }^{22-26}$ Another big challenge is that for most existing PSs, it is quite difficult to manipulate their targets by merely adjusting the external conditions without changing their molecular structure. Thus, the target manipulation of a single PS without introducing a specific targeting group is worth exploring and is of great research significance. Based on the aforementioned situation, it is highly challenging but rewarding to develop a simple AIE-based PS with six conspicuous merits including: (1) facile preparation and stable chemical structure, (2) moderate water solubility, (3) high ROS efficiency with bright emission, (4) good biocompatibility, (5) high specificity to targets and (6) low dark toxicity but significant light toxicity. More importantly, the achievement of multiple yet successive targeting in one individual structure-fixed PS is scarcely reported. That is to say: under a given condition, the PS can only recognize one specific target. However, by varying the condition, the target changes correspondingly. Thus, achieving the success to this target-manipulation by varying the external conditions in one individual PS not only opens a new door for PS design principle but also widens the applications for defending against pathogens and cancer.

In this contribution, motivated by anion- $\pi^{+}$interactions on triggering AIE effect, ${ }^{27,28}$ we designed and prepared an AIEbased PS, namely 4TPA-BQ, with such interactions via one step synthesis. The inherent positive charge endows the molecule with modest water solubility for bioapplications. Simultaneously, 4TPA-BQ shows aggregation-induced ROS generation (AIROSG) effect, with a ${ }^{1} \mathrm{O}_{2}$ quantum yield of up to unity. Unprecedentedly, time-dependent PDT for multiple targets was achieved by controlling the incubation time of 4TPA-BQ with varied targets. In the presence of white light irradiation for $15 \mathrm{~min}$, 4TPA-BQ could eliminate drug-resistant Escherichia coli infection efficiently, leaving host tissues unaffected. When the incubation time was lengthened to $12 \mathrm{~h}$,
4TPA-BQ could kill cancer cells with slight harm to normal cells (Scheme 1).

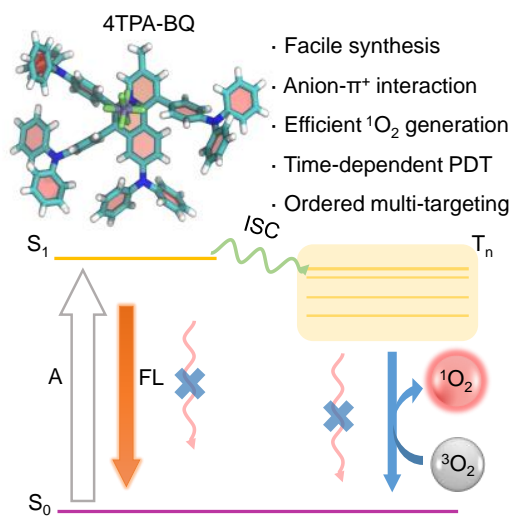

Scheme 1. Illustration of 4TPA-BQ for time-dependent PDT.

\section{RESULTS AND DISCUSSION}

The synthetic route of 4TPA-BQ was depicted in Figure 1A, 4,4'-(1,2-ethynediyl)bis[N,N-diphenylbenzenamine] (1) was synthesized via a modified Sonogashira coupling reaction according to the reported literature (Scheme S1) preliminarily, ${ }^{29}$ then a facile one-pot reaction of compound $\mathbf{1}$ and 2 methylallylamine produced 4TPA-BQ with a satisfactory yield of $81 \%{ }^{30}$ Detailed experimental procedures were provided in the Supporting Information. Both compound $\mathbf{1}$ and the product were fully characterized by ${ }^{1} \mathrm{H}$ NMR, ${ }^{13} \mathrm{C}$ NMR and highresolution mass spectroscopies (Figure S1-S6). In addition, the structure of TPA-BQ was directly certified by the single crystal $\mathrm{X}$-ray diffraction analysis.

After confirming the structure of 4TPA-BQ, we investigated its optical properties. As shown in Figure S7, its UV-vis absorption spectrum presents two broad peaks at the wavelength of $375 \mathrm{~nm}$ and $440 \mathrm{~nm}$ in DMSO solution. Then photoluminescence (PL) spectrum was measured in DMSO/water mixtures to study its AIE property. As presented in Figure 1B, 1C and Table 1 , when water fraction $\left(f_{\mathrm{w}}\right)$ is below $40 \%$, the PL intensity is quite weak with negligible fluorescence quantum yield $\left(\Phi_{\text {Soln }}=\right.$ $0.30 \%$ ) and nearly no emission was observed by naked eyes under a hand-held UV light irradiation. However, upon increasing $f_{\mathrm{w}}$ to $50 \%$, the emission intensity amplified sharply. An observation of bright orange fluorescence $\left(\Phi_{\text {Aggr. }}=6.70 \%\right)$ was obtained at $f_{\mathrm{w}}=70 \%$, in which emission intensity enhanced to about 40-fold compared to that of DMSO solution. In addition, formation of aggregates in poor solvents confirmed by dynamic light scattering (DLS) results (Figure S8) suggests the typical AIE feature of 4TPA-BQ. The red-shifted emission (from 490 $\mathrm{nm}$ to $594 \mathrm{~nm}$ ) with increased $f_{\mathrm{w}}$ is likely due to the twisted intramolecular charge transfer (TICT) effect, which is common for D-A structure molecules. Compared to the orange emission of forming aggregates, the solids exhibit a stronger and blueshifted emission $\left(\Phi_{\text {Solid }}=15.30 \%\right)$, which is ascribed to the closely and ordered molecular packing, testified by powder Xray diffraction (PXRD) analysis (Figure S9). In contrast to no obvious diffraction peaks observed for aggregates, the PXRD pattern of solids displayed two sharp peaks at $4.9^{\circ}$ and $19.5^{\circ}$, indicating the crystalline nature of solids. Further, we calculated and compared the radiative decay rate $\left(k_{r}\right)$ and nonradiative decay rate $\left(k_{n r}\right)$ in different states. The $k_{r}$ increased from $0.07 \times$ $10^{7} \mathrm{~s}^{-1}$ in solution to $1.19 \times 10^{7} \mathrm{~s}^{-1}$ in aggregate state, while the $k_{n r}$ decreased from $22.74 \times 10^{7} \mathrm{~s}^{-1}$ to $16.64 \times 10^{7} \mathrm{~s}^{-1}$. Although 
the $k_{n r}$ of solid $\left(27.28 \times 10^{7} \mathrm{~s}^{-1}\right)$ was close to that of solution state $\left(22.74 \times 10^{7} \mathrm{~s}^{-1}\right)$, yet the $k_{r}$ of solid powder $\left(4.93 \times 10^{7} \mathrm{~s}^{-1}\right)$ rose by two orders of magnitude. This accounts for the AIE characteristic of 4TPA-BQ.
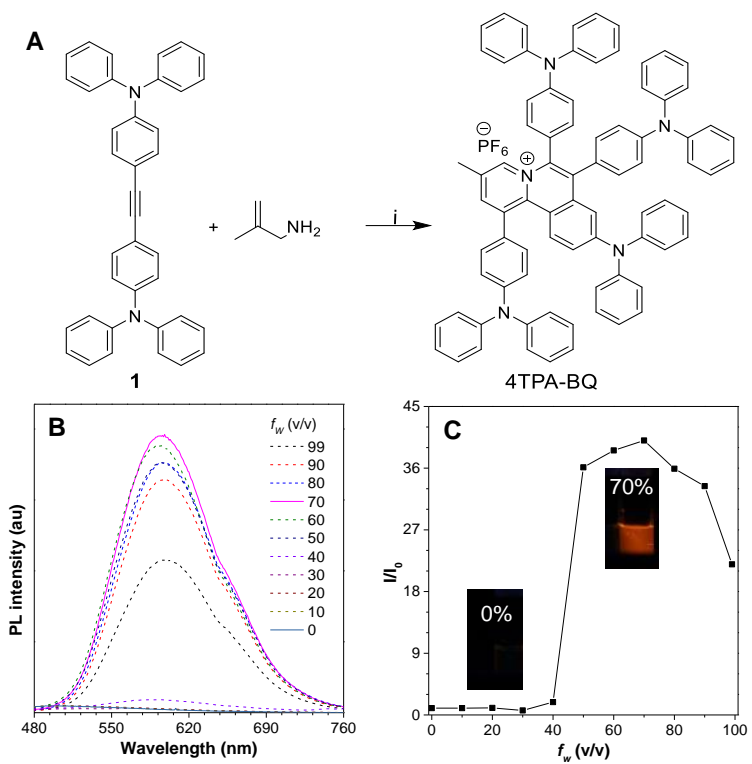

Figure 1. (A) Synthetic route to 4TPA-BQ: (i) $\mathrm{NaPF}_{6},\left[\mathrm{Cp} * \mathrm{RhCl}_{2}\right]_{2}$, $\mathrm{Cu}(\mathrm{OAc})_{2} \mathrm{H}_{2} \mathrm{O}, \mathrm{MeOH}, 130{ }^{\circ} \mathrm{C}, 6 \mathrm{~h}$. (B) PL spectra of 4TPA-BQ in $\mathrm{DMSO} /$ water mixtures with different water fractions $\left(f_{\mathrm{w}}\right)$. (C) Plot of the relative emission intensity $\left(\mathrm{I} / \mathrm{I}_{0}\right)$ versus $f_{\mathrm{W}}$. Inset: fluorescence images of 4TPA-BQ in solution $\left(f_{\mathrm{W}}=0 \%\right)$ and aggregate $\left(f_{\mathrm{W}}\right.$ $=70 \%$ ) states under irradiation from a hand-held UV lamp. Excitation wavelength: $380 \mathrm{~nm}$; Concentration: $10 \mu \mathrm{M}$.

Table 1. Photophysical properties of 4TPA-BQ.

\begin{tabular}{llll}
\hline & Solution & Aggregate & Solid \\
\hline$\lambda_{e x}(\mathrm{~nm})$ & 490 & 594 & 570 \\
$\Phi(\%)$ & 0.30 & 6.70 & 15.30 \\
$\tau(\mathrm{ns})^{a}$ & 4.39 & 5.61 & 3.10 \\
$k_{r}\left(\times 10^{7} \mathrm{~s}^{-1}\right)^{b}$ & 0.07 & 1.19 & 4.93 \\
$k_{n r}\left(\times 10^{7} \mathrm{~s}^{-1}\right)^{c}$ & 22.74 & 16.64 & 27.28 \\
\hline
\end{tabular}

${ }^{a} \tau$ is defined as average fluorescence lifetime calculated by $\tau=$ $\Sigma \mathrm{A}_{i}\left(\tau_{i}\right)^{2} / \Sigma \mathrm{A}_{i} \tau_{i}$, where $\mathrm{A}_{i}$ is the pre-exponential factor for lifetime $\tau_{i}$. ${ }^{b} k_{r}=\Phi / \tau .{ }^{c} k_{n r}=1 / \tau-k_{r}$.

For in-depth understanding of the AIE feature of 4TPA-BQ, we obtained its single crystal in chloroform/hexane mixtures by slow evaporation. It is noticed that anion- $\pi^{+}$interactions between the fluorine atoms of hexafluorophosphate anions and the positively charged benzoquinoline core were found with the distance of $3.063 \AA$ and calculated energy of $-61.68 \mathrm{~kJ} / \mathrm{mol}$, which impedes $\pi-\pi$ stacking in the crystal to avoid emission quenching (Figure 2A). Intramolecular and intermolecular hydrogen bonds of F-H interactions were also found in the crystal lattice, constraining rotation of the phenyl rings effectively, rigidifying structure in the crystal lattice (Figure 2A and 2C). Besides, the large dihedral angles between TPA moieties and benzoquinoline core were observed as $64.37^{\circ}\left(\theta_{1}\right), 82.61^{\circ}\left(\theta_{2}\right)$ and $66.49^{\circ}\left(\theta_{3}\right)$, further preventing detrimental $\pi-\pi$ interactions (Figure $2 \mathrm{~B}$ and $\mathrm{S} 10)$. Consequently, anion $-\pi^{+}$interactions together with highly twisted molecular conformation favors AIE characteristic of 4TPA-BQ, which agrees well with previously reported literature. $^{28}$

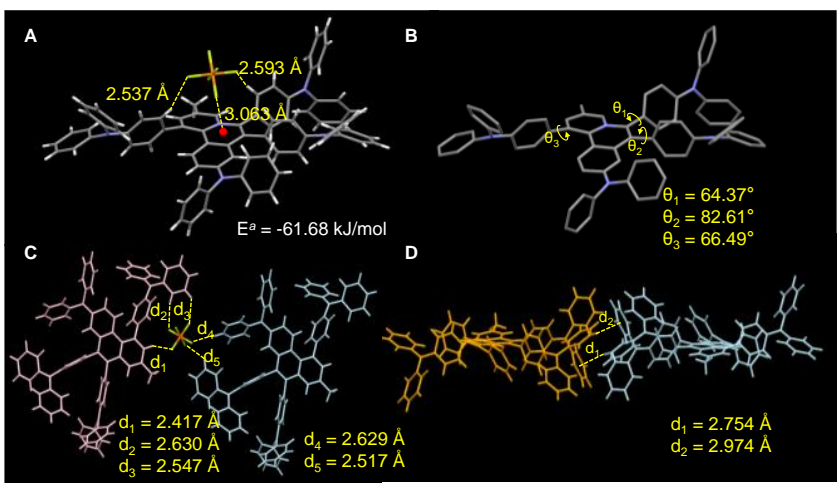

Figure 2. (A) Anion- $\pi^{+}$and F-H interactions, (B) dihedral angles, (C) intermolecular hydrogen bonding and (D) $\mathrm{C}-\mathrm{H} \cdots \pi$ interactions in the single crystal structure of 4TPA-BQ. ${ }^{a} \mathrm{E}$ is defined as interaction energy between $\pi^{+}$and anion calculated based on the singlecrystal structure by single-point calculations using M062X/6$31+\mathrm{G}(\mathrm{d}, \mathrm{p})$ method.

Considering its typical D-A structure in favor of ${ }^{1} \mathrm{O}_{2}$ generation, ${ }^{31}$ preliminary experiments were performed to explore ${ }^{1} \mathrm{O}_{2}$ generation ability of 4TPA-BQ. Here, N,N'-di(2,3dihydroxypropyl)-9,10-anthracenedipropanamide (DHPA) was utilized as ${ }^{1} \mathrm{O}_{2}$ indicator for the reason that (1) it can detect ${ }^{1} \mathrm{O}_{2}$ specifically as DHPA will yield endoperoxide under ${ }^{3} \mathrm{O}_{2}$ oxidation, leading to an obvious decrease in absorbance; (2) it has no interaction with cationic PSs, thus this non-ionic chemical trap can evaluate ${ }^{1} \mathrm{O}_{2}$ production in a more accurate way. ${ }^{32}$ Firstly, we measured the UV-vis absorption spectra of DHPA in 4TPA-BQ aggregates and solution under white light irriadition, respectively (Figure S11). As presented in Figure 3A, it is worth noticing that the absorbance of DHPA decreased sharply in the presence of 4TPA-BQ aggregates, whereas irradiation on 4TPA-BQ solution did not lead to obvious DHPA consumption. This can be explained by that, on one hand, formation of aggregates can induce excited states energy splitting, thus promoting ISC rate; ${ }^{33}$ on the other, according to RIM mechanism, aggregates will minimize the competitive nonradiative decay, which favors ISC process. Thus, AIE feature can activate ${ }^{1} \mathrm{O}_{2}$ generation, which can be defined as AIROSG. Commercial available PSs including Ce6 and Rose Bengal were also compared under the same circumstances (Figure S11). After 8 min exposure to white light, compared to more than $60 \%$ DHPA consumption in the presence of 4TPABQ aggregates, only $10 \%$ and 50\% DHPA degraded in Ce6 and Rose Bengal, respectively (Figure 3A). The results reveal that 4TPA-BQ aggregates can generate ${ }^{1} \mathrm{O}_{2}$ more efficiently than $\mathrm{Ce} 6$ and Rose Bengal, both of which are commonly used and high ${ }^{1} \mathrm{O}_{2}$ production PSs for PDT. ${ }^{34,35}$ Furthermore, taking DHPA as an indicator and Rose Bengal as the standard PS, we calculated ${ }^{1} \mathrm{O}_{2}$ quantum yield of 4TPA-BQ in aqueous medium following the reported procedure. ${ }^{36}$ As exhibited in Figure $3 \mathrm{~B}$ and Figure S12, a high value of $97.8 \%$ was gained. From the above results, it is suggested that 4TPA-BQ in aqueous medium can produce ${ }^{1} \mathrm{O}_{2}$ at a high efficiency, rendering it a promising candidate for PDT application. For elucidation of its extremely high ${ }^{1} \mathrm{O}_{2}$ quantum yield, theoretical calculations were performed based on time-dependent density functional theory (TD-DFT) via Gaussian 09. As indicated in Figure 3C, notably, 
the energy gap between $\mathrm{S}_{1}$ and $\mathrm{T}_{4}$ state is only $0.03 \mathrm{eV}$, which is much smaller compared to most reported PSs. ${ }^{37,38}$ Apparently, for 4TPA-BQ, it is the sufficiently narrow $\triangle E_{S T}$ rather than SOC that plays the dominant role in enhancing ISC rate as well as high ${ }^{1} \mathrm{O}_{2}$ generation efficiency. Successively, natural transition orbitals (NTOs) were calculated for excited states analysis. As shown in Figure 3D, the hole NTOs are mainly localized on TPA moieties while the electron NTOs are mainly localized on benzoquinoline core. Thus, the highly separated charge distribution realized by incorporation of electron donor (TPA moieties) and acceptor (benzoquinoline core) contributes to the narrow energy gap between singlet and triplet states. ${ }^{39-41}$ Furthermore, to manifest the existence of triplet excited states which is pivotal for producing ${ }^{1} \mathrm{O}_{2}$, we performed the nanosecond transient absorption experiments (Figure S13). The determined triplet lifetime of 4TPA-BQ aggregates was $81 \mathrm{~ns}$ while no signal was detected for 4TPA-BQ in solution state, which again validates that formation of aggregates favors ISC process.
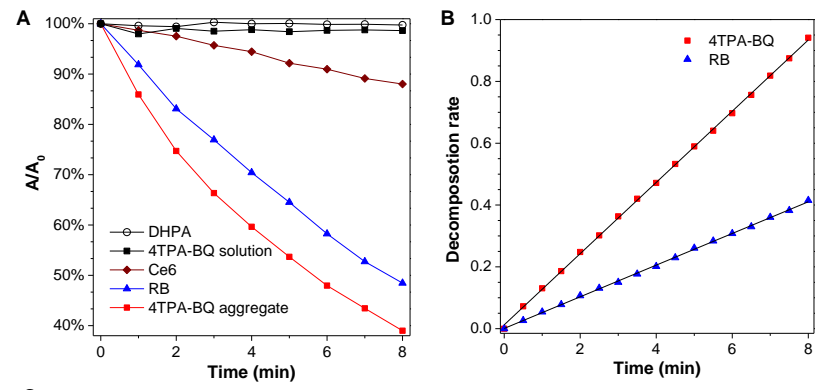

C
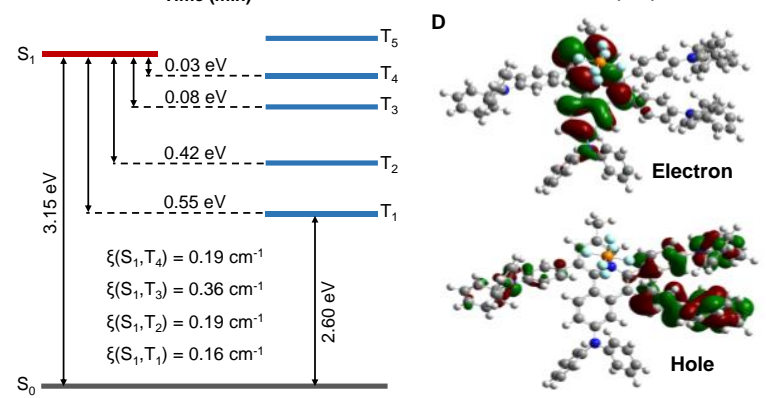

Figure 3. (A) Plot of relative absorbance of DHPA without and with different PSs under white light irradiation, where $\mathrm{A}_{0}$ and $\mathrm{A}$ are the absorbance of DHPA at $378 \mathrm{~nm}$ before and after white light irradiation, respectively. $[\mathrm{PS}]=9 \times 10^{-6} \mathrm{M},[\mathrm{DHPA}]=4.5 \times 10^{-5} \mathrm{M}$. (B) Decomposition rate of DHPA with 4TPA-BQ aggregates and $\mathrm{RB}$, respectively. (C) Calculated energy level diagram between singlet and triplet states. (D) The natural transition orbitals (NTOs) of 4TPA-BQ.

Since 4TPA-BQ is positively charged, it is expected to target both bacteria and cells via electrostatic interactions. ${ }^{42}$ Here, confocal laser scanning microscopy (CLSM) was utilized to verify the assumptions. The ubiquitous and well-studied Staphylococcus epidermidis (S. epidermidis) and Escherichia coli $(E$. coli) were selected as representatives for Gram-positive and Gram-negative bacteria, respectively. As illustrated in Figure 4, the bright orange fluorescence from 4TPA-BQ treated $E$. coli and $S$. epidermidis was readily observed with high contrast to background, indicating that 4TPA-BQ can bind to both gram-negative and gram-positive bacteria efficiently. Subsequently, normal mammalian cells including COS-7 and HLF cells were incubated with 4TPA-BQ under the same conditions (Figure S14). Unexpectedly, scarcely no fluorescence signals can be detected under CLSM for these two kinds of cells, hinting that 4TPA-BQ cannot target normal mammalian cells. Thus, the bacterial and cell imaging results indicate that 4TPA-BQ has the potential for selectively recognizing and imaging bacteria over normal cells.

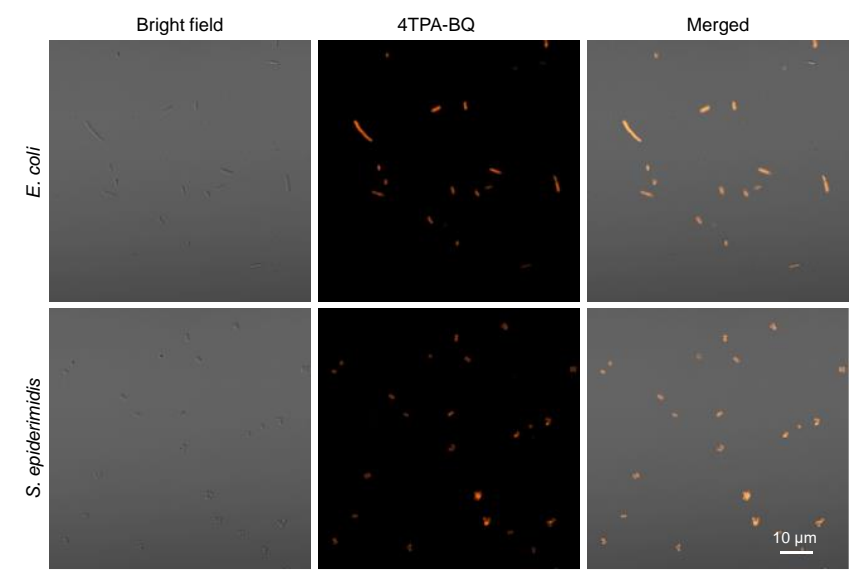

Figure 4. CLSM images of E. coli and S. epiderimidis incubated with $5 \mu \mathrm{M}$ 4TPA-BQ for 15 min.

Inspired by the specific bacterial-targeting capability and high ${ }^{1} \mathrm{O}_{2}$ generation yield of 4TPA-BQ, we would like to study and compare its antibacterial activity and cytotoxicity towards normal cells, which were evaluated by plate count method and MTT assay, respectively. As presented in Figure 5A and 5B, 4TPA-BQ displays a dose-dependent antibacterial efficiency towards both $E$. coli and $S$. epidermidis under white light illumination. About $90 \%$ E. coli and S. epidermidis were killed at a low concentration of $2 \mu \mathrm{M}$; and when 4TPA-BQ reached 5 $\mu \mathrm{M}$, almost no bacterial colony could be observed on the agar for both $E$. coli and S. epidermidis (Figure 5D). Meanwhile, for groups incubated without irradiation, no obvious drop of survival rate was found with increased concentration of 4TPA$B Q$, which suggests that the potent antimicrobial activity of 4TPA-BQ is entirely due to its intrinsic ${ }^{1} \mathrm{O}_{2}$ generation induced by white light irradiation. Considering its high killing efficiency on $E$. coli and $S$. epidermidis, we tried drug-resistant bacterial strains, ampicillin-resistant E. coli. As shown in Figure 5C, less than $30 \%$ ampicillin-resistant $E$. coli survived when treated with $5 \mu \mathrm{M}$ 4TPA-BQ under white light irradiation. When increasing to $10 \mu \mathrm{M}$, the killing efficiency reached to almost $100 \%$. Similarly, no dark toxicity to ampicillin-resistant $E$. coli was found. Therefore, when in the absence of irradiation, 4TPA-BQ can visualize bacteria in situ; When in the presence of irradiation, efficient ${ }^{1} \mathrm{O}_{2}$ generation ability endows 4TPA-BQ with powerful antibacterial capability, even including drugresistant bacteria. To acquire solid evidence of its antibacterial activity, SEM analysis was conducted to visualize morphological changes of E. coli and S. epidermidis (Figure S15). For control groups, regular body shape and intact cell walls could be clearly observed. Similar results were also achieved for groups treated with 4TPA-BQ but no light. By contrast, for bacteria treated with both 4TPA-BQ and light, the walls were rigorously damaged, collapsed and fused. These direct information from SEM is in consistent with the antibacterial results. Subsequently, we investigated the toxicity of 4TPA-BQ towards normal cells. As illustrated in Figure 5E, for both dark and illumination groups, no obvious drop of COS7 cell viability was observed with increased 4TPA-BQ concentration. It is worth noticing that a highly active cell 
viability of $91 \%$ was maintained even under the high concentration of $10 \mu \mathrm{M}$, in sharp contrast to the antibacterial results. Analogous results were also achieved for HLF cells (Figure 5F). MTT results reveals that 4TPA-BQ displays excellent biocompatibility with normal cells. Thus, 4TPA-BQ can cause fatal damage to bacteria but negligible cytotoxicity to normal cells.
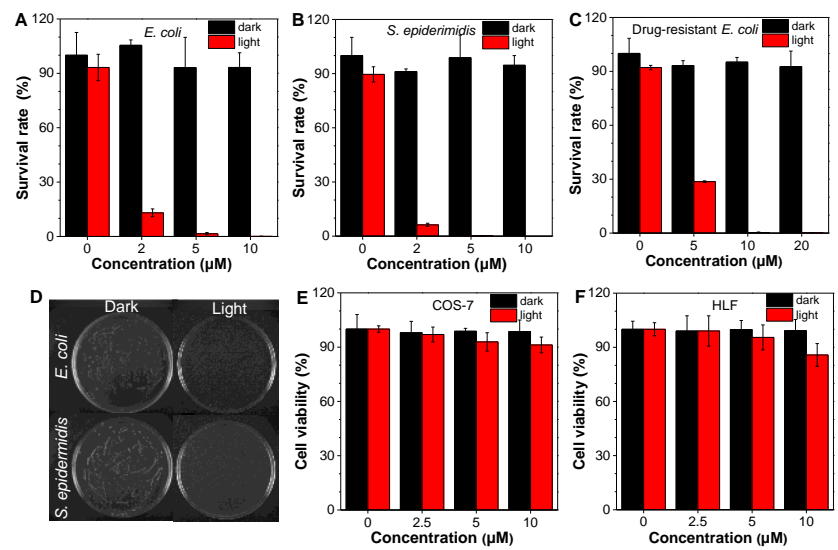

Figure 5. (A-C) Killing efficiency of 4TPA-BQ on E. coli, $S$. epiderimidis and ampicillin-resistant E. coli. (D) Photographs of $E$. coli and S. epidermidis cultured on agar plate supplemented with 5 $\mu \mathrm{M}$ 4TPA-BQ. (E-F) Cell viability of COS-7 and HLF cells incubated with 4TPA-BQ for $15 \mathrm{~min}$.

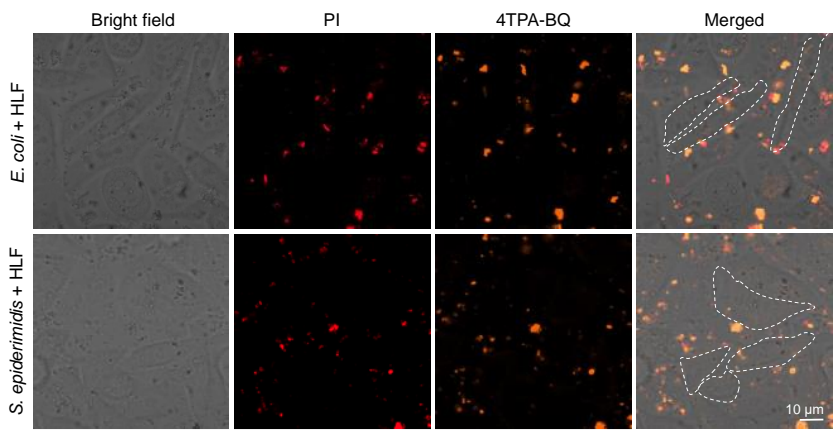

Figure 6. CLSM images of E. coli and HLF cells and $S$. epiderimidis and HLF cells stained with PI after incubation with 5 $\mu \mathrm{M}$ 4TPA-BQ under white light irradiation. The dash lines indicate HLF cells.

To further testify that 4TPA-BQ can realize the selective bacterial-targeting over normal cells, we cultured $E$. coli or $S$. epidermidis mixed with mammalian cells in the presence of 4TPA-BQ and light irradiation. Here, propidium iodide (PI) was utilized to mark the dead bacteria or cells if any. As shown in Figure 6 and S16, the detected red fluorescence of PI and orange fluorescence of 4TPA-BQ was from bacteria, whereas hardly no fluorescence was from mammalian cells, indicating that 4TPA-BQ killed the bacteria efficiently while normal cells were still alive. Hence, the co-culture experiments fully validated that 4TPA-BQ is competent to specifically and selectively recognize and kill bacteria upon light irradiation with normal mammalian cells essentially unaffected.

In order to gain deep insight to selective bacterial targeting mechanism, zeta potential measurements, which offered the information of surface charges of bacteria and cells, were performed. The acquired data was summarized in Table S2. For untreated groups, the zeta potentials of E. coli $(-19.55 \pm 2.53 \mathrm{mV})$ and $S$. epidermidis $(-16.53 \pm 1.40 \mathrm{mV})$ are more negative than
COS-7 cells $(-13.08 \pm 0.32 \mathrm{mV})$ and HLF cells $(-10.90 \pm 0.96$ $\mathrm{mV}$ ), suggesting that selective binding is actuated by stronger electrostatic interactions with bacteria. After treated with 4TPA-BQ, the zeta potentials of cells and $S$. epidermidis barely changed, while E. coli (from $-19.55 \pm 2.53 \mathrm{mV}$ to $-9.75 \pm 0.85 \mathrm{mV}$ ) become more positively surface charged. The unchanged surface charges of COS-7 (from $-13.08 \pm 0.32 \mathrm{mV}$ to $13.18 \pm 1.02 \mathrm{mV}$ ) and HLF (from $-10.90 \pm 0.96 \mathrm{mV}$ to $10.35 \pm 2.32 \mathrm{mV}$ ) cells revealed there are no interactions with 4TPA-BQ. The different zeta potential changes of $E$. coli and $S$. epidermidis are related to their cell wall architecture. ${ }^{43}$ For Gram-positive $S$. epidermidis, it has a thick but porous outer peptidoglycan wall and negatively charged teichoic acids inserting in the thick wall, thus 4TPA-BQ can be almost held in the pores without exposure to surface, leading to subtle change on $S$. epidermidis surface charge. As for Gram-negative E. coli, an additional membrane layer composed by negatively charged lipopolysaccharides (LPS) is located outside the peptidoglycan layer, making 4TPA-BQ stay on the surface, thus the surface charges of $E$. coli become more positive. The zeta potential data is in accordance with bacterial and cell imaging results. Therefore, we proposed a possible explanation for selective bacterial targeting. (1) The driving force for selective recognition is electrostatic interaction. Though both bacteria and cells bear negative charges on their surface, bacteria are more negatively charged, thus the positively charged 4TPA-BQ target bacteria in priority. (2) Considering the short lifetime $(<0.04 \mu \mathrm{s})$ and limited radius of action $(<0.02 \mu \mathrm{m})$ of ${ }^{1} \mathrm{O}_{2}$ as well as the size of mammalian cells $(>20 \mu \mathrm{m}),{ }^{14}$ the damage on mammalian cells is imperceptible even under the situation that a spot of 4TPA-BQ enter into cells.

Encouraged by its desired bacterial targeting and eliminating performance in vitro, we further evaluated the antibacterial ability of 4TPA-BQ against drug-resistant bacteria in vivo. Third-degree burns, also known as full thickness burns, are the most serious burns, involving the whole skin layer damage, skin necrosis and eschar formation. The recovery of wounds is highly challenging. Thus, we established a third-degree burn wounds infected with Ampicillin-resistant E. coli on mice model ${ }^{44}$ After burn and infection, they were randomly assigned to 3 groups, treated with PBS only (Control group), 4TPA-BQ only (Dark group) and 4TPA-BQ with white light irradition (PDT group), respectively. The macroscopic appearance of wound healing process was recorded at different time points. As shown in Figure 7A and 7B, at day 3 and 5 post burn, ulceration and purulence appeared on the wound surface in both Dark and Control groups, while the PDT group showed no such signs. At day 8, the sizes of Ampicillin-resistant $E$. coli infected wounds in the PDT group were significantly smaller in comparison to the other two groups. At day 10, the wounds in the PDT group had completely recovered with newly regenerated skins, while the wounds in the other two groups were relatively large with pus under the scab after uncovering. At day 5 and 10 post burn, we took the damaged skin tissues for bacterial culture and count (Figure 7C, Figure S17 and S18A). It turned out that the number of bacteria in the PDT group was significantly less than that in the Control group and the Dark group. Similarly, at day 5 post burn, the liver and spleen were ground for bacteria counting. As presented in Figure 7C and S18B, there were almost no colonies in the organs from PDT group, displaying a sharp contrast to the dark and control groups. This result indicates that the PDT treatment had excellent germicidal efficacy and can effectively inhibit further infection of bacteria from the skin into deep 
organs. ${ }^{45}$ In addition, we further evaluated the wound healing efficacy of sectioned tissues by hematoxylin and eosin (H\&E) staining (Figure 7D). ${ }^{46}$ At day 5, the intact and thick epidermis, as well as the hair follicles and blood vessels, were clearly observed in the neonatal skin of the PDT group. The neonatal structure recovered completely in the PDT group at day 10 . However, in the Dark and Control groups, no epidermis appeared and almost no new hair follicles and blood vessels were observed at day 5 and 10 . Therefore, the in vivo experiments successfully demonstrated that 4TPA-BQ has excellent antimicrobial effect under white light irradiation that can significantly promote the wound healing process, consistent with in vitro tests.
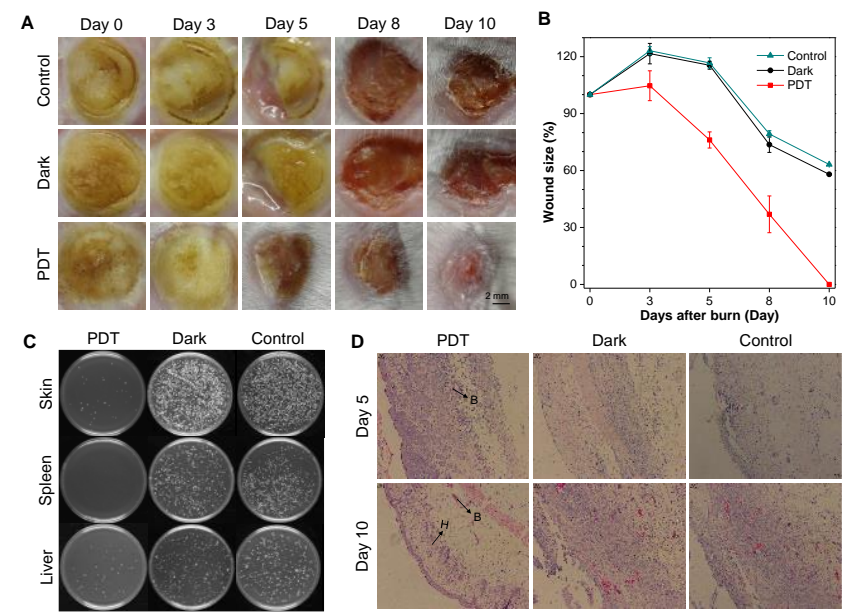
Control

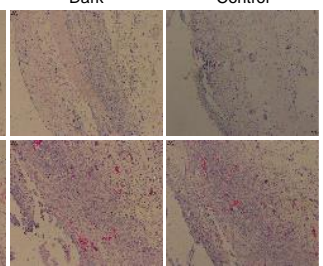

Figure 7. Antibacterial activity against ampicillin-resistant $E$. coli infection in vivo. (A) Photographs of bacterial infected mice wounds treated with PBS only (Control group), 4TPA-BQ only (Dark group) and 4TPA-BQ+white light irradiation (PDT group), respectively. (B) Changes of the infected wound size subjected to different treatments. (C) Plate photographs of bacterial amount of skin wound and organs at day 5 in 100 -fold dilution. (D) Hemotoxylin and eosin stain of the infected skin slices of day 5 and day 10. B: blood vessel; $\mathrm{H}$ : hair folliculus.
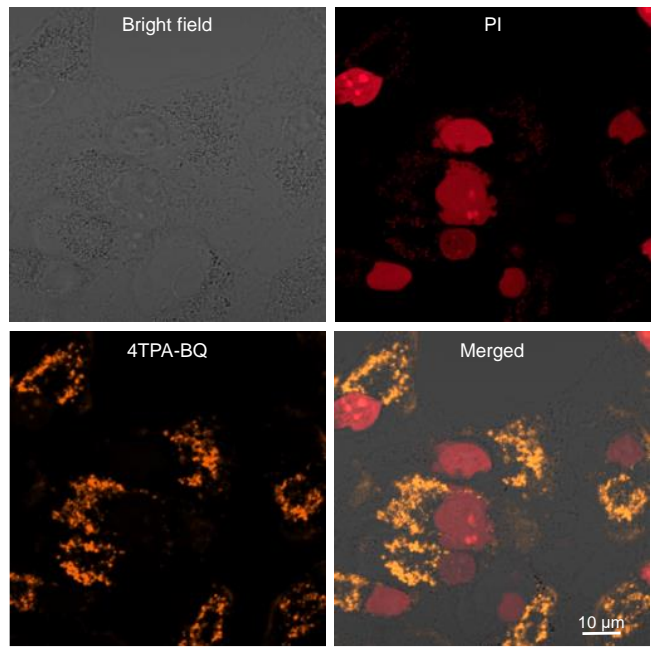

Figure 8. CLSM images of HeLa cells stained with PI upon incubation with $10 \mu \mathrm{M}$ 4TPA-BQ for $12 \mathrm{~h}$ under white light irradiation.

So far, the study appears finished here, still we want to go deeper into the interaction between the positively charged PS and the negatively charged cells, and to see whether the interaction can be manipulated by regulating external conditions. Given the aforementioned cell staining time is only $15 \mathrm{~min}$, we would like to see whether prolong staining time will make any difference (Figure S19). When COS-7 normal cells treated with 4TPA-BQ for $4 \mathrm{~h}$, almost no fluorescence signals could be detected, similar with CLSM images of $15 \mathrm{~min}$. When extending incubation time to $8 \mathrm{~h}$, weak orange florescence was observed. And it was found that much more 4TPA-BQ accumulated into COS-7 cells after $12 \mathrm{~h}$ incubation. Thus, 4TPA-BQ can accumulate into normal cells under the sufficient long incubation time, however, the fluorescence intensity is too weak for effective visualization. Inspired by the previous report that cancer cells are more negatively surface charged than normal cells, HeLa cancer cells were utilized for cellular imaging under the same conditions. ${ }^{47}$ Intensified fluorescence of 4TPA-BQ was observed along with incubation time as well. Notably, in comparison to COS-7 cells, fluorescence of HeLa cells is remarkably stronger and clearer, hinting that the positively charged PS target cancer cells preferentially over normal cells. Taking advantage of its preference to cancer cells and efficient ${ }^{1} \mathrm{O}_{2}$ generation, both normal and cancer cells were employed for PDT application. As shown in Figure S20 and Figure 8, the CLSM images of 4TPA-BQ is consistent with those of cellular imaging. For PI channel, no fluorescence was detectable at $4 \mathrm{~h}$ for both COS-7 and HeLa cells; After $8 \mathrm{~h}$ incubation, faint red fluorescence from HeLa cells could be noticed whereas COS-7 cells remained no red fluorescence; when incubation time further extended to $12 \mathrm{~h}$, nearly all HeLa cancer cells in sight were stained with PI with enhanced red fluorescence, in sharp contrast to COS-7 cells. The combination of cellar imaging and PDT application reveals that when regulating incubation time to a sufficient long period, 4TPABQ can eliminate cancer cells with no apparent toxicity towards normal cells through PDT process.

As demonstrated by above results, a time-dependent PDT towards varying targets was obtained by manipulating incubation time of PS. Virtually, cooperation of electrostatic attraction and hydrophobic effect plays a decisive role in this process. ${ }^{48,49}$ On the one hand, electrostatic interaction serves as the driving force for possible recognition. The positively charged 4TPA-BQ can target bacteria and cells in sequence, due to the fact that the absolute value of negative surface charges is ranked as bacteria>cancer cells $>$ normal cells. ${ }^{42,47}$ That is, the more negative the target is charged, the easier it can be bound to the positively charged PS. On the other, in the respect of differentiating bacteria and cells, hydrophobic 4TPA-BQ with $\mathrm{C} \log \mathrm{P}=14$ tends to target bacteria preferentially than cells. ${ }^{50,51}$ Consequently, cationic charge and hydrophobicity is well balanced in 4TPA-BQ. The positive charge endows it with modest water solubility desired for bio application, and more significantly, it is the prerequisite for recognizing varying targets including microbes and cells. Additionally, electrostatic interaction and hydrophobicity makes the multi-targeting in sequence. Essentially, it is the reasonable molecular structure design that realizes the multiple as well as ordered targeting therapeutic effect, which provide guidelines for the future development of antibacterial and anticancer materials.

\section{CONCLUSIONS}

In summary, a simple AIE-based phototherapeutic agent, namely TPA-BQ, with anion- $\pi^{+}$interactions was designed and achieved successfully via one step synthesis. The inherent 
anion- $\pi^{+}$interactions as well as the highly twisted conformation are responsible for its AIE feature, verified by single crystal analysis. An extremely high ${ }^{1} \mathrm{O}_{2}$ quantum yield of $98.7 \%$ was obtained in aggregate form thanks to the aggregation-induced ROS generation (AIROSG) effect, overwhelmingly surpassing commercial PSs. Theoretical calculations suggested that sufficient small $\Delta \mathrm{E}_{S T}$ plays a dominate role in the ISC process, desired for ${ }^{1} \mathrm{O}_{2}$ generation. Prominently, 4TPA-BQ can bind to different targets by controlling incubation time. A broadspectrum and potent antibacterial activity was attained after incubating 4TPA-BQ with pathogens for $15 \mathrm{~min}$. Upon light irradiation, it targeted and killed both Gram-negative and Gram-positive bacteria at a low concentration of $2 \mu \mathrm{M}$, leaving normal cells unaffected. Both in vitro and in vivo experiments demonstrated its outstanding bacterial eliminating performance against ampicillin-resistant $E$. coli infection. When incubated with cells for $12 \mathrm{~h}$, 4TPA-BQ specifically killed cancer cells with low toxicity to normal cells via PDT process. We proposed that this time-dependent PDT was achieved by collaboration of electrostatic attraction and hydrophobic effect between 4TPABQ and the corresponding targets. To the best of our knowledge, it is the first reported target-controllable PDT achieved by adjusting external conditions instead of changing the PS structure. Future work will focus on shifting the PS emission to near-infrared region. Therefore, the readily accessible strategy not only provides a platform for next generation PS, but also offers new insights for clinical applications of synergizing with conventional therapeutic modalities.

\section{ASSOCIATED CONTENT}

Supporting Information. Materials and methods, synthetic procedures, characterization, crystallographic data and computational details, including Figures S1-S20 and Tables S1-S2. (PDF). This material is available free of charge via the Internet at http://pubs.acs.org.

\section{AUTHOR INFORMATION}

\section{Corresponding Author}

*wangjg@iccas.ac.cn

*tangbenz@ust.hk

\section{ORCID}

Jianguo Wang: 0000-0003-0984-9716

Ben Zhong Tang: 0000-0002-0293-964X

\section{Author Contributions}

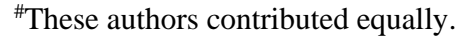

\section{Notes}

The authors declare no competing financial interest.

\section{ACKNOWLEDGMENT}

This work was partially supported by the National Natural Science Foundation of China Grant (21871060 and 81271476), the University Grants Committee of Hong Kong (AoE/P-03/08), the Innovation and Technology Commission (ITC-CNERC14SC01 and ITCRD/17-9), the Research Grants Council of Hong Kong (16308016, C6009-17G and A-HKUST605/16), and the Science and Technology Plan of Shenzhen (JCYJ20160229205601482).

\section{REFERENCES}

(1) Nolivos, S.; Cayron, J.; Dedieu, A.; Page, A.; Delolme, F.; Lesterlin, C. Science 2019, 364, 778.
(2) Siegel, R. L.; Miller, K. D.; Jemal, A. CA A Cancer J Clin. 2019 69,7 .

(3) Ng, C. W.; Li, J.; Pu, K. Adv. Funct. Mater. 2018, 28, 1804688.

(4) Zhen, X.; Zhang, J.; Huang, J.; Xie, C.; Miao, Q.; Pu, K. Angew. Chem. Int. Ed. 2018, 57, 7804.

(5) Al-Afyouni, M. H.; Rohrabaugh, T. N.; Al-Afyouni, K. F.; Turro, C. Chem. Sci. 2018, 9, 6711.

(6) Gao, Y.; Wang, X.; He, X.; He, Z.; Yang, X.; Tian, S.; Meng, F.; Ding, D.; Luo, L.; Tang, B. Z. Adv. Funct. Mater. O, 1902673.

(7) Shi, H.; Ma, X.; Zhao, Q.; Liu, B.; Qu, Q.; An, Z.; Zhao, Y.; Huang, W. Adv. Funct. Mater. 2014, 24, 4823.

(8) Tao, D.; Feng, L.; Chao, Y.; Liang, C.; Song, X.; Wang, H.; Yang, K.; Liu, Z. Adv. Funct. Mater. 2018, 28, 1804901.

(9) Yuan, H.; Chong, H.; Wang, B.; Zhu, C.; Liu, L.; Yang, Q.; Lv, F.; Wang, S. J. Am. Chem. Soc. 2012, 134, 13184.

(10) Dougherty, T. J.; Gomer, C. J.; Henderson, B. W.; Jori, G.; Kessel, D.; Korbelik, M.; Moan, J.; Peng, Q. J. Natl. Cancer Inst. 1998, 90, 889.

(11) Klymchenko, A. S. Acc. Chem. Res. 2017, 50, 366.

(12) Lovell, J. F.; Liu, T. W. B.; Chen, J.; Zheng, G. Chem. Rev. 2010, 110, 2839.

(13) He, X.; Xiong, L.-H.; Zhao, Z.; Wang, Z.; Luo, L.; Lam, J. W. Y.; Kwok, R. T. K.; Tang, B. Z. Theranostics 2019, 9, 3223.

(14) Hu, F.; Xu, S.; Liu, B. Adv. Mater. 2018, 30, 1801350.

(15) Zhu, C.; Kwok, R. T. K.; Lam, J. W. Y.; Tang, B. Z. ACS Appl. Bio Mater. 2018, 1, 1768.

(16) Luo, J.; Xie, Z.; Lam, J. W. Y.; Cheng, L.; Chen, H.; Qiu, C.; Kwok, H. S.; Zhan, X.; Liu, Y.; Zhu, D.; Tang, B. Z. Chem. Commun. 2001, 1740.

(17) Alifu, N.; Dong, X.; Li, D.; Sun, X.; Zebibula, A.; Zhang, D.; Zhang, G.; Qian, J. Mater. Chem. Front. 2017, 1, 1746.

(18) Huang, Y.; Zhang, G.; Hu, F.; Jin, Y.; Zhao, R.; Zhang, D. Chem. Sci. 2016, 7, 7013.

(19) Wang, M.; Zhang, G.; Zhang, D.; Zhu, D.; Tang, B. Z. J. Mater. Chem. 2010, 20, 1858

(20) Gao, H.; Zhang, X.; Chen, C.; Li, K.; Ding, D. Adv. Biosys. 2018, 2, 1800074.

(21) Mei, J.; Leung, N. L.; Kwok, R. T.; Lam, J. W.; Tang, B. Z. Chem. Rev. 2015, 115, 11718.

(22) Feng, G.; Zhang, C. J.; Lu, X.; Liu, B. ACS Omega 2017, 2, 546.

(23) Gao, M.; Yu, F.; Lv, C.; Choo, J.; Chen, L. Chem. Soc. Rev. 2017, 46, 2237.

(24) Huang, W.-C.; Tsai, P.-J.; Chen, Y.-C. Nanomedicine 2007, 2, 777.

(25) Li, X.; Kim, J.; Yoon, J.; Chen, X. Adv. Mater. 2017, 29 , 1606857.

(26) Li, X.; Robinson, S. M.; Gupta, A.; Saha, K.; Jiang, Z.; Moyano, D. F.; Sahar, A.; Riley, M. A.; Rotello, V. M. ACS Nano 2014, 8, 10682

(27) Wang, J.; Gu, X.; Ma, H.; Peng, Q.; Huang, X.; Zheng, X.; Sung, S. H. P.; Shan, G.; Lam, J. W. Y.; Shuai, Z.; Tang, B. Z. Nat. Commun. 2018, 9, 2963.

(28) Wang, J.; Gu, X.; Zhang, P.; Huang, X.; Zheng, X.; Chen, M.; Feng, H.; Kwok, R. T. K.; Lam, J. W. Y.; Tang, B. Z. J. Am. Chem. Soc. 2017, 139, 16974.

(29) Chen, Z.-W.; Zhu, Y.-Z.; Ou, J.-W.; Wang, Y.-P.; Zheng, J.Y. J. Org. Chem. 2014, 79, 10988.

(30) Han, Y. R.; Shim, S.-H.; Kim, D.-S.; Jun, C.-H. Org. Lett. 2017, 19, 2941

(31) Xu, S.; Yuan, Y.; Cai, X.; Zhang, C.-J.; Hu, F.; Liang, J.; Zhang, G.; Zhang, D.; Liu, B. Chem. Sci. 2015, 6, 5824.

(32) Martinez, G. R.; Garcia, F.; Catalani, L. H.; Cadet, J.; Oliveira, M. C. B.; Ronsein, G. E.; Miyamoto, S.; Medeiros, M. H. G.; Mascio, P. D. Tetrahedron 2006, 62, 10762.

(33) Yang, L.; Wang, X.; Zhang, G.; Chen, X.; Zhang, G.; Jiang, J. Nanoscale 2016, 8, 17422

(34) Meng, Z.; Yu, B.; Han, G.; Liu, M.; Shan, B.; Dong, G.; Miao, Z.; Jia, N.; Tan, Z.; Li, B.; Zhang, W.; Zhu, H.; Sheng, C.; Yao, J. J. Med. Chem. 2016, 59, 4999.

(35) Paczkowski, J.; Lamberts, J. J. M.; Paczkowska, B.; Neckers, D. C. Free Radic. Biol. Med. 1985, 1, 341. 
(36) Wang, D.; Su, H.; Kwok, R. T. K.; Hu, X.; Zou, H.; Luo, Q.; Lee, M. S.; Xu, W.; Lam, J. W. Y.; Tang, B. Z. Chem. Sci. 2018.

(37) Wu, W.; Mao, D.; Hu, F.; Xu, S.; Chen, C.; Zhang, C.-J.; Cheng, X.; Yuan, Y.; Ding, D.; Kong, D.; Liu, B. Adv. Mater. 2017, 29, 1700548.

(38) Xu, S.; Wu, W.; Cai, X.; Zhang, C.-J.; Yuan, Y.; Liang, J.; Feng, G.; Manghnani, P.; Liu, B. Chem. Commun. 2017, 53, 8727.

(39) Endo, A.; Sato, K.; Yoshimura, K.; Kai, T.; Kawada, A.; Miyazaki, H.; Adachi, C. Appl. Phys. Lett. 2011, 98, 083302.

(40) Uoyama, H.; Goushi, K.; Shizu, K.; Nomura, H.; Adachi, C. Nature 2012, 492, 234.

(41) Schmidt, K.; Brovelli, S.; Coropceanu, V.; Beljonne, D.; Cornil, J.; Bazzini, C.; Caronna, T.; Tubino, R.; Meinardi, F.; Shuai, Z.; Brédas, J.-L. J. Phys. Chem. A 2007, 111, 10490.

(42) Zhu, C.; Yang, Q.; Liu, L.; Lv, F.; Li, S.; Yang, G.; Wang, S. Adv. Mater. 2011, 23, 4805.

(43) Maisch, T.; Szeimies, R.-M.; Jori, G.; Abels, C. Photochem. Photobiol. Sci. 2004, 3, 907.

(44) Bunyapraphatsara, N.; Jirakulchaiwong, S.; Thirawarapan, S.; Manonukul, J. Phytomedicine 1996, 2, 247.
(45) Zhao, M.; Yang, M.; Baranov, E.; Wang, X.; Penman, S.; Moossa, A. R.; Hoffman, R. M. Proc. Natl. Acad. Sci. U.S.A. 2001, 98 , 9814.

(46) Li, Y.; Wang, S.; Huang, R.; Huang, Z.; Hu, B.; Zheng, W.; Yang, G.; Jiang, X. Biomacromolecules 2015, 16, 780.

(47) Chen, B.; Le, W.; Wang, Y.; Li, Z.; Wang, D.; Ren, L.; Lin, L.; Cui, S.; Hu, J. J.; Hu, Y.; Yang, P.; Ewing, R. C.; Shi, D.; Cui, Z. Theranostics 2016, 6, 1887.

(48) Liu, G.-j.; Tian, S.-n.; Li, C.-y.; Xing, G.-w.; Zhou, L. ACS Appl. Mater. Interfaces 2017, 9, 28331.

(49) Palermo, E. F.; Lienkamp, K.; Gillies, E. R.; Ragogna, P. J. Angew. Chem. 2019, 131, 3728-3731.

(50) Horobin, R. W.; Rashid-Doubell, F.; Pediani, J. D.; Milligan, G. Biotech Histochem 2013, 88, 440.

(51) Phillips, R. L.; Miranda, O. R.; You, C.-C.; Rotello, V. M.; Bunz, U. H. F. Angew. Chem. Int. Ed. 2008, 47, 2590. 
Table of Contents

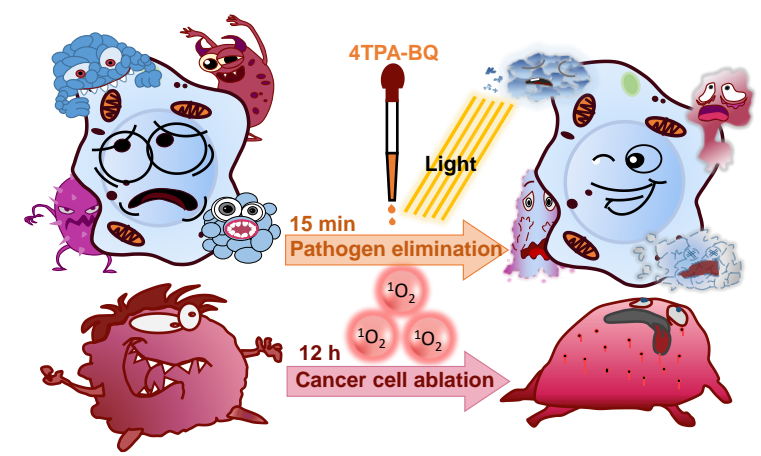

